\title{
Report of 2 Novel Presentations of Subcutaneous Fat Necrosis of the Newborn
}

\author{
Susanna M.H. Kannenberg ${ }^{a} \quad$ H. Francois Jordaan ${ }^{a}$ Willem I. Visser ${ }^{a}$ \\ Fatima Ahmed $^{\mathrm{b}}$ A. Fourie Bezuidenhout ${ }^{\mathrm{c}}$ \\ a Division of Dermatology, Department of Medicine, Stellenbosch University and Tygerberg \\ Academic Hospital, Cape Town, South Africa; ${ }^{b}$ Dermatology Department, Tawam Hospital, \\ Al Ain City, United Arab Emirates; ${ }^{c}$ Department of Radiology, Beth Israel Deaconess Medical \\ Centre, Harvard Medical School, Boston, MA, USA
}

\section{Keywords}

Fat necrosis · Newborn · Abscess-like plaques · Haemorrhage

\section{Abstract}

Subcutaneous fat necrosis of the newborn (SCFNN) is a rare form of panniculitis classically affecting healthy full-term infants. There are a number of predisposing factors including perinatal asphyxia. The condition generally has a benign course with spontaneous resolution, but monitoring for metabolic complications, in particular the potentially life-threatening complication of hypercalcaemia, is critical. The authors report 2 cases of preterm infants with perinatal asphyxia with atypical presentations of SCFNN: the first with bony involvement resembling Langerhans cell histiocytosis and with follicular pseudocarcinomatous hyperplasia on histology; and the second presenting with a huge haematoma requiring surgical debridement. Both cases were initially erroneously diagnosed as pyogenic infections.

\section{Introduction}

Subcutaneous fat necrosis of the newborn (SCFNN) typically occurs in full-term neonates during the first month of life [1,2], but in the more recent literature, premature infants are reported more frequently. Possible precipitating factors include hypothermia, hypogly- 
caemia, perinatal hypoxaemia, and birth trauma [1-3]. We describe 2 cases of SCFNN with hitherto undescribed associations: pseudocarcinomatous hyperplasia originating from follicular epithelium permeated by neutrophils and bony involvement simulating Langerhans cell histiocytosis (LCH) (case 1); and a massive haematoma necessitating surgical drainage (case 2). Of further interest is that both cases were premature neonates with perinatal asphyxia and initially misdiagnosed as pyogenic infections.

\section{Case Report}

\section{Case 1}

A male was delivered at 35 weeks by emergency caesarean section. The baby suffered perinatal asphyxia and developed hypoxic ischaemic encephalopathy (HIE). In his third week of life, he developed abscess-like plaques on his scalp and left ankle, and the patient was commenced on intravenous antibiotics. Incision and drainage revealed culture-negative purulent material. One week later, a dermatology referral and radiographic imaging was requested due to non-resolution of the plaques. Radiography of the left lower extremity showed a localized area of subcutaneous soft tissue thickening with focal, irregular and amorphous calcification medial to the left ankle. Magnetic resonance imaging (MRI) of the head showed multiple areas of subcutaneous scalp fat swelling with abnormal hypointense signal change (Fig. 1a). The left occipito-parietal lesion showed infiltrative margins and involved the underlying outer table of the skull and diploic space, with sparing of the inner table of the skull (Fig. 1a). All lesions demonstrated avid contrast enhancement on MRI (Fig. 1b). The clinical picture combined with the scull findings suggested a differential diagnosis of LCH, tuberculosis, tertiary syphilis and neuroblastoma metastases. A skin biopsy of the scalp, however, revealed subcutaneous fat necrosis with the exceptional finding of folliculocentric pseudocarcinomatous hyperplasia (Fig. 1c-e). He was managed conservatively, and the plaques had resolved completely at the 4-month follow-up. At 18 months of age, the patient was diagnosed with the uncommon Sotos syndrome (cerebral gigantism) based on clinical findings and genetic analysis. Of note, bone involvement simulating LCH and an increased susceptibility to developing SCFNN have not been documented in Sotos syndrome.

Case 2

A preterm (36 weeks' gestation) male was born by caesarean section for severe foetal distress. He developed severe HIE, did not fulfil the criteria for therapeutic cooling and was therefore managed conservatively. During his second week of life, the neonatologists noticed an indurated red plaque on his back and diagnosed cellulitis. Despite intravenous antibiotics, the plaques did not abate. A dermatology opinion was sought when a rapidly enlarging dark red tumour developed in the plaque (Fig. 2a). A clinical diagnosis of SCFNN was made complicated by a large haematoma. Surgical debridement (Fig. 2b) of the haemorrhagic mass was performed, and tissue was sent for histology. The presence of a large haematoma was confirmed. Scattered necrotic adipocytes with numerous needle-shaped spaces were seen amongst the erythrocytes (Fig. 2c) confirming the clinical diagnosis of SCFNN. The baby had an uncomplicated post-operative stay, and the SCFNN plaques resolved gradually with residual scarring. 


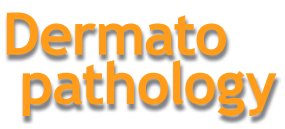

\begin{tabular}{l|l}
\hline Dermatopathology 2019;6:147-152 \\
\hline DOI: 10.1159/000497176 & $\begin{array}{l}\text { @ } 2019 \text { The Author(s). Published by S. Karger AG, Basel } \\
\text { www.karger.com/dpa }\end{array}$ \\
\hline
\end{tabular}

Kannenberg et al.: Report of 2 Novel Presentations of Subcutaneous Fat Necrosis of the Newborn
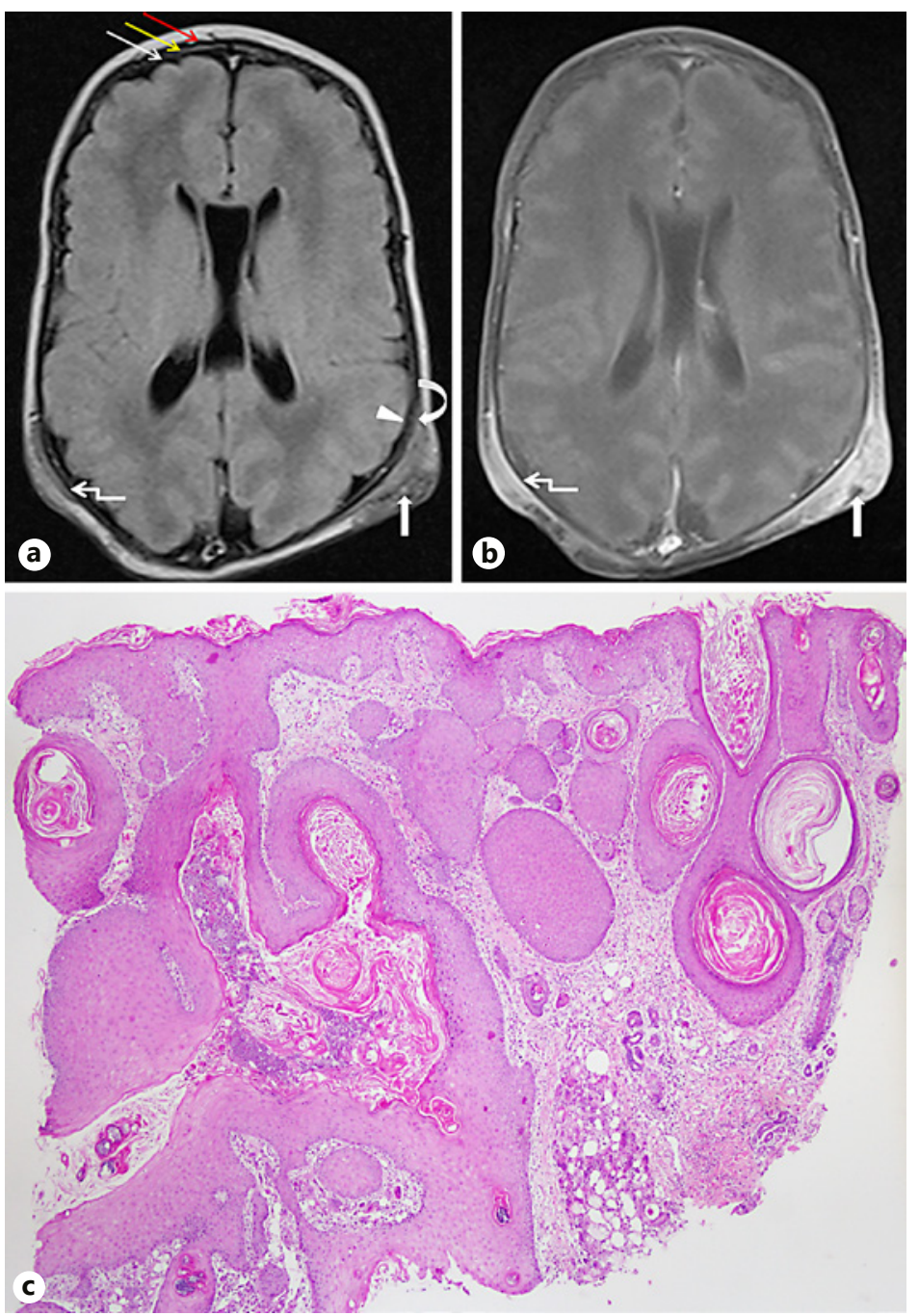

Fig. 1. a Axial FLAIR MR image demonstrates swelling and decreased signal intensity of the involved subcutaneous scalp fat in the left occipito-parietal (bold straight arrow) and right occipito-parietal (crooked arrow) areas. There is involvement of the underlying outer table of the skull and diploic space (curved arrow) with preservation of the inner table of the skull (arrowhead) in the left occipito-parietal area. Compare with normal trilaminar appearance of the skull in the right frontal area on FLAIR MR image: hypointense outer table (red arrow), isointense diploic space (yellow arrow) and hypointense inner table (thin white arrow). b Post-contrast T1 fat-saturated MR image demonstrating avid contrast enhancement in the left occipitoparietal (bold straight arrow) and right occipito-parietal (crooked arrow) areas of swollen subcutaneous fat. c Subcutaneous fat necrosis with folliculocentric pseudocarcinomatous hyperplasia and acute and chronic inflammation. Brown-Hopps gram and PAS with diastase stains were negative for bacteria and fungi, respectively. H\&E. $\times 100$. d Medium magnification of $\mathbf{c}$ with typical needle-shaped clefts within lipocytes and granulomatous infiltrate. Note adjacent pseudocarcinomatous hyperplasia. H\&E. $\times 200$. e Higher magnification of the involved area depicted in c and $\mathbf{d}$. The needle-shaped clefts in lipocytes, foamy histiocytes and an associated foreign body granulomatous infiltrate are clearly visualized. H\&E. $\times 400$.

(Figure continued on next page.) 

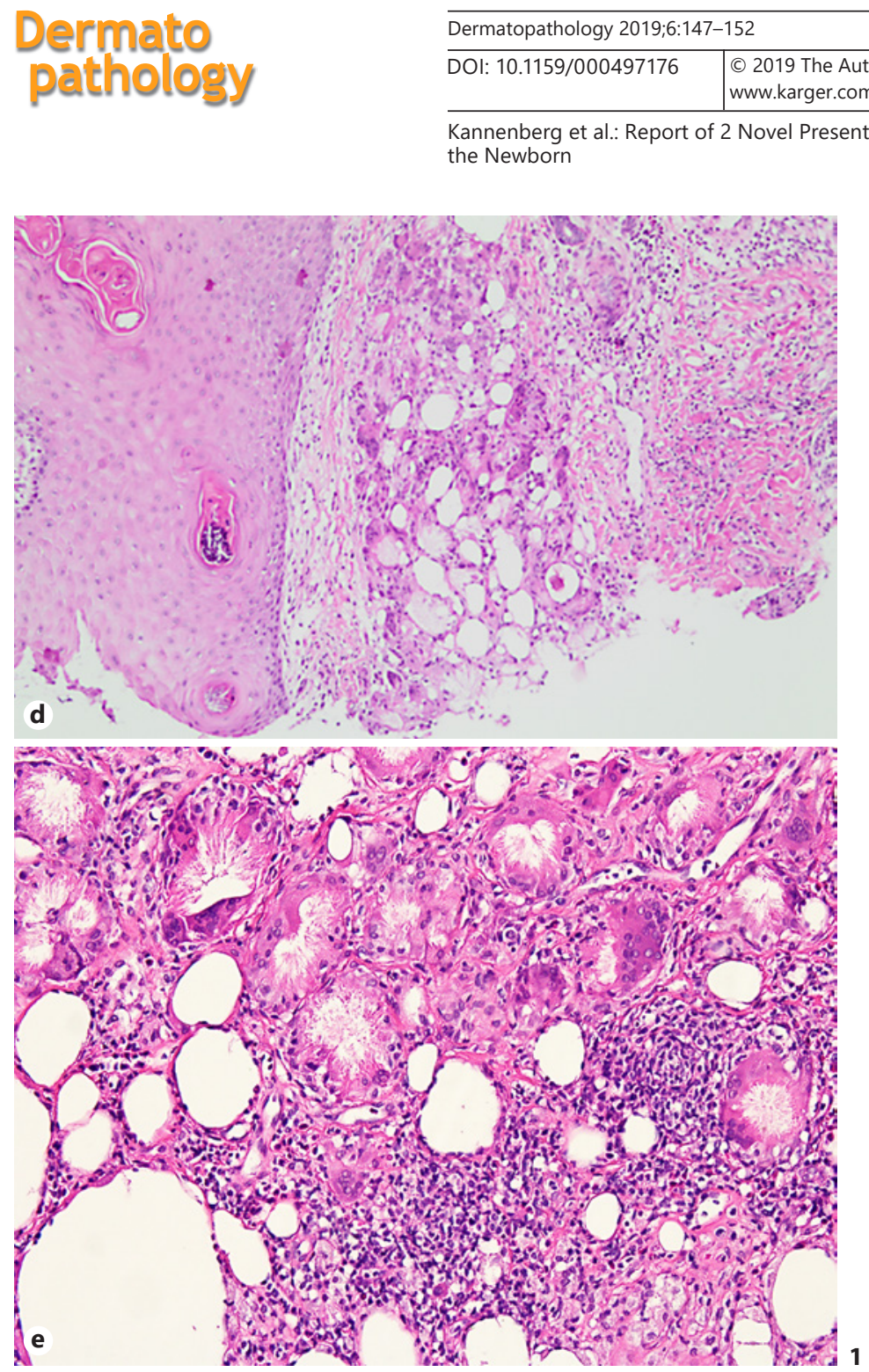

\section{Discussion/Conclusion}

SCFNN typically occurs in full-term neonates during the first month of life. Possible precipitating factors include hypothermia (as with therapeutic cooling for HIE [4]), hypoglycaemia, perinatal hypoxaemia and, possibly, birth trauma [1-3]. The exact mechanism remains unclear [1], but it is believed that the high ratio of saturated to unsaturated fatty acids, in some way, results in crystallization, adipocyte injury and granulomatous inflammation.

The condition presents as characteristically symmetrical, circumscribed, erythematous, indurated subcutaneous nodules and plaques favouring the cheeks, shoulders, back, buttocks and thighs. Although the plaques are usually not ulcerated, a single case of SCFNN plaques ulcerating was described in a full-term baby [5].

Microscopic examination shows a lobular panniculitis with neutrophils, lymphocytes and macrophages, as well as needle-shaped clefts in a radial array in lipocytes, macrophages and giant cells. Foci of calcification may be present. The crystals are doubly refractile and stain with Oil Red 0. Needle-shaped clefts may be absent. The epidermis is usually not involved. Fine-needle aspiration of the subcutaneous lesions has been reported as a possible alternative to skin biopsy for the diagnosis, revealing the typical needle-shaped clefts [6]. 

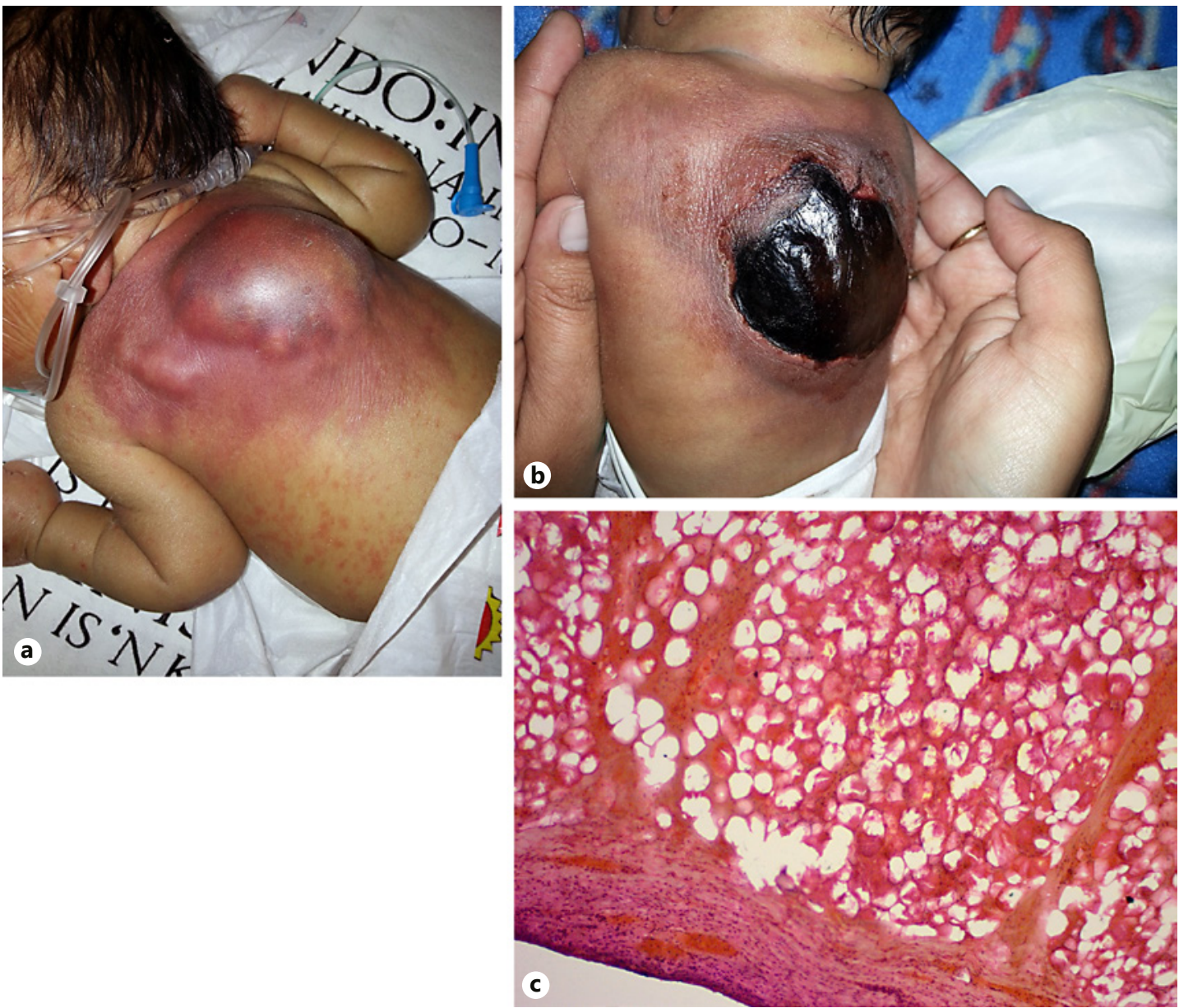

Fig. 2. a Neonate with large purple tumour on the background of SCFNN. Note surrounding petechiae; the patient had thrombocytopaenia. b Haematoma visible during surgical debridement. c Scattered necrotic adipocytes with numerous needle-shaped spaces seen amongst the erythrocytes. H\&E. $\times 100$.

Spontaneous resolution in the first year of life is the rule, and management is usually supportive. Systemic corticosteroids may be indicated in severe cases. Local complications of SCFNN include lipoatrophy and ulceration, but they very rarely require surgery. A single case requiring surgery due to non-resolution of plaques was reported by Beuzeboc Gérard et al. [7] in 2014. Systemic complications include thrombocytopaenia, hypertriglyceridaemia and hypercalcaemia, which may be fatal [1]. The true incidence of hypercalcaemia as a complication is unknown and appears on average 6 months after fat necrosis. Infants with hypercalcaemia may remain asymptomatic, may present with non-specific features, such as poor feeding and hypotonia, or may even present with systemic dystrophic calcifications, such as nephrolithiasis. The exact duration of monitoring for hypercalcaemia remains unclear. Hypercalcaemia is managed by hydration, dietary restriction of calcium and vitamin D, calcium-wasting diuretics (e.g., furosemide), calcitonin, bisphosphonates (e.g., etidronate) [8] and systemic corticosteroids.

Sclerema neonatorum is the main condition in the differential diagnosis of SCFNN. This is usually a fatal disorder, with widespread panniculitis in toxically ill premature infants. Clinically, the condition is characterized by wax-like hardening of the whole skin sparing the palms and soles. The histopathologic findings consist of extensive fibrosis in the subcutaneous fat. 
The authors report 2 unique cases, thereby expanding the clinical spectrum of SCFNN. Both cases were initially misdiagnosed as pyogenic infections. In case 1 , bone involvement simulating LCH was identified as a novel finding. The usually uninvolved epidermis showed folliculocentric pseudocarcinomatous hyperplasia. Case 2 was complicated by a massive haematoma and required surgical debridement, which is also unique in the usually uncomplicated course of SCFNN.

In conclusion, the authors affirm that SCFNN also affects premature neonates and may follow perinatal asphyxia. SCFNN may involve bony structures when it mimics LCH, may be misdiagnosed as pyogenic infections, may be complicated by massive haematomas and may occasionally require surgery. Histology may also rarely demonstrate follicular pseudocarcinomatous hyperplasia.

\section{Statement of Ethics}

The authors have no ethical conflicts to disclose. The parents gave informed consent.

\section{Disclosure Statement}

The authors have no conflicts of interest to declare.

\section{Funding Sources}

This manuscript was not sponsored.

\section{Author Contributions}

S.M.H.K. and H.F.J. conceived the idea. S.M.H.K., H.F.J., F.A. and A.F.B. collected the data. S.M.H.K., H.F.J. and A.F.B. prepared the manuscript, and all authors were responsible for critical revision of the manuscript.

\section{References}

1 Mitra S, Dove J, Somisetty SK. Subcutaneous fat necrosis in newborn-an unusual case and review of literature. Eur J Pediatr. 2011 Sep;170(9):1107-10.

2 Mahé E, Girszyn N, Hadj-Rabia S, Bodemer C, Hamel-Teillac D, De Prost Y. Subcutaneous fat necrosis of the newborn: a systematic evaluation of risk factors, clinical manifestations, complications and outcome of 16 children. Br J Dermatol. 2007 Apr;156(4):709-15.

3 Burden AD, Krafchik BR. Subcutaneous fat necrosis of the newborn: a review of 11 cases. Pediatr Dermatol. 1999 Sep-Oct;16(5):384-7.

4 Del Pozzo-Magaña BR, Ho N. Subcutaneous Fat Necrosis of the Newborn: A 20-Year Retrospective Study. Pediatr Dermatol. 2016 Nov;33(6):e353-5.

5 Fenniche S, Daoud L, Benmously R, Ben Ammar F, Khelifa I, Chaabane S, et al. Subcutaneous fat necrosis: report of two cases. Dermatol Online J. 2004 Oct;10(2):12.

6 Schubert PT, Razack R, Vermaak A, Jordaan HF. Fine-needle aspiration cytology of subcutaneous fat necrosis of the newborn: the cytology spectrum with review of the literature. Diagn Cytopathol. 2012 Mar;40(3):245-7.

7 Beuzeboc Gérard M, Aillet S, Bertheuil N, Delliere V, Thienot S, Watier E. Surgical management of subcutaneous fat necrosis of the newborn required due to a lack of improvement: a very rare case. Br J Dermatol. 2014 Jul; 171(1):183-5.

8 Pérez Martínez E, Camprubí Camprubí M, Ramos Cebrián M, Antón López J, Apodaca Saracho A, Lopez Ramos $\mathrm{MG}$, et al. Treatment with bisphosphonates in severe hypercalcemia due to subcutaneous fat necrosis in an infant with hypoxic-ischemic encephalopathy. J Perinatol. 2014 Jun;34(6):492-3. 